\title{
Screening and validation of differentially expressed extracellular miRNAs in acute pancreatitis
}

\author{
SHISHUAI MENG ${ }^{1}$, HAO WANG ${ }^{2}$, DONGBO XUE $^{2}$ and WEIHUI ZHANG ${ }^{2}$ \\ Departments of ${ }^{1}$ Critical Care Medicine and ${ }^{2}$ General Surgery, The First Affiliated Hospital \\ of Harbin Medical University, Harbin, Heilongjiang 150001, P.R. China
}

Received October 21, 2016; Accepted June 8, 2017

DOI: $10.3892 / \mathrm{mmr} .2017 .7374$

\begin{abstract}
The present study aimed to screen for differentially expressed extracellular microRNAs (miRNAs) during the development of acute pancreatitis (AP) and validate the miRNA expression in the plasma of patients with AP. The culture medium of taurolithocholic acid-3 sulfate-treated rat pancreatic acinar AR42J cells was collected to extract total RNA for miRNA microarray analysis. Compared with the miRNA test results of the AP rats in the GEO databases, the differentially expressed extracellular miRNAs were screened. The TargetScan, miRanda, and PicTar programs were used for target gene prediction of the identified miRNAs, and gene ontology-biological processes (GO-BP) functional annotation was performed. Finally, the results from the combined microarray analyses (in vitro cell line and in vivo rat samples) were validated using plasma samples from patients with mild and moderately severe AP by reverse transcription-polymerase chain reaction. The results demonstrated that extracellular miR-24 was differentially expressed by microarray and bioinformatics analysis in both the cell line and the animal model of AP. Bioinformatics prediction analysis revealed that downstream target genes of miR-24 included Vav2, Syk, Lhcgr, Slc9a3r1, Cacnb1, Cacna1b, Bcl10, and Fgd3. Functional enrichment analysis revealed that the main GO-BP predicted functional presentations were positive regulation of calcium-mediated signaling, activation of c-Jun $\mathrm{N}$-terminal kinase activity, calcium ion transport, regulation of Rho protein signal transduction, negative regulation of the protein kinase $B$ signaling cascade, and the $\mathrm{T}$ cell receptor signaling pathway. Validation analysis for the plasma miR-24 expression in humans revealed a significant upregulation of miR-24 in the plasma samples of AP patients compared with the healthy controls, while no
\end{abstract}

Correspondence to: Dr Dongbo Xue, Department of General Surgery, The First Affiliated Hospital of Harbin Medical University, 23 YouZheng Street, Harbin, Heilongjiang 150001, P.R. China

E-mail: xue9971@sina.com

Key words: acute pancreatitis, distant organ injury, circulating microRNAs, anti-inflammatory, modular analysis significant difference was observed in the miR-24 expression between the mild and the moderately severe AP groups. The present study confirmed the high expression of miR-24 in peripheral blood during AP, suggesting that miR-24 might have an intercellular communication role contributing to the AP-associated distant organ injury.

\section{Introduction}

Acute pancreatitis (AP) is a common critical illness in the general surgery department and intensive care unit (ICU). AP can develop rapidly and cause multiple organ dysfunction syndrome and even endanger lives (1). AP is associated with long-term drinking, increased triglyceride levels, and mechanical pancreatic duct obstruction. The development of AP is usually attributed to stimulation of the pancreas by excessive production, release, or early activation of proteolytic enzymes (2). Injured acinar cells release inflammatory mediators to activate immune cells and induce inflammatory cell infiltration, thus promoting the development and aggravation of $\operatorname{AP}(3,4)$.

Severe AP usually causes functional injury of distant organs, especially lung injury. Multiple studies have indicated that the mechanism underlying AP-induced lung injury may be associated with excessive release of cytokines and inflammatory mediators, cell apoptosis, and excessive accumulation of neutrophils and macrophages (5). During the induction of distant organ dysfunction by AP through intercellular and interorgan signaling, reactive oxygen species might be a key factor $(6,7)$. However, the specific mechanism and the processes in the inflammatory reactions and injury of distant organs induced by AP remain unclear.

The present study evaluated the extracellular microRNA (miRNA) expression profiles of the culture medium from activated pancreatic acinar cells and of mesenteric lymph samples from an AP animal model, using microarray analysis. The differential miRNA expression was then validated in plasma samples from patients with AP, in order to provide evidence for potentially novel therapeutic targets in the treatment of AP.

\section{Materials and methods}

Measurement of miRNAs in the culture medium of activated AR42J cells 
Cell experiments and grouping. Rat pancreatic acinar AR42J cells were purchased from the China Center for Type Culture Collection (Wuhan, China). The AR42J cell line was cultured in DMEM (Gibco; Thermo Fisher Scientific, Inc., Waltham, MA, USA) containing 10\% fetal bovine serum (Gibco; Thermo Fisher Scientific, Inc.), $100 \mathrm{U} / \mathrm{ml}$ penicillin and $100 \mu \mathrm{g} / \mathrm{ml}$ streptomycin in a $37^{\circ} \mathrm{C}$ and $5 \% \mathrm{CO}_{2}$ incubator (8).

AR42J cells were divided into two groups: The control group did not receive any treatment, while the experimental group was treated with $200 \mu \mathrm{M}$ TLC-S for $40 \mathrm{~min}$. Following replacement with fresh medium, the cells were then cultured for an additional $2 \mathrm{~h}$ prior to collection of the culture medium.

Microarray detection and data analysis. Total RNA from culture medium was extracted using a Plasma/Serum Circulating and Exosomal RNA Purification kit (cat. no. 42800; Norgen Biotek Corp., Thorold, ON, Canada) according to the manufacturer's instructions. Quality control was performed using reverse transcription-quantitative polymerase chain reaction (RT-qPCR) on has-miR-16 and has-miR-192 (9). According to the recommended $\mathrm{Ct}$ value range of indicators in a literature report, the extracted total RNA was determined to have sufficient quality for use in the subsequent microarray experiments (9). The microarray platform used was a rat $\mu$ Paraflo microRNA microarray assay (LC Sciences, Houston, TX, USA). The negative control demonstrated a lower hybridization signal value than the background signal value. It was concluded that the microarray assay met the quality standards. Labeling and hybridization experiments were performed according to the microarray manufacturer's instructions. The hybridization images were collected using a laser scanning-based system (GenePix 4000B; Molecular Devices LLC, Sunnyvale, CA, USA), and digital conversion of images was performed using the Array-Pro Analyzer image analysis software (Media Cybernetics, Inc., Rockville, MD, USA). For data analysis, the background value was first subtracted and then data were normalized using LOWESS (Media Cybernetics) (2), and the locally weighted regression. miRNAs with a fold change $<0.5$ or $>2$ were considered differentially expressed miRNAs. $\mathrm{P}<0.01$ was used as the threshold value for differentially expressed miRNAs (10).

\section{Detection of miRNAs in the mesenteric lymph of AP rats} Source of gene microarray data. The gene expression profile data were downloaded from the Gene Expression Omnibus database (National Center for Biotechnology Information, http://www.ncbi.nlm.nih.gov/geo) with the series number GSE42455. In the original publication by Blenkiron et al, intraductal infusion of sodium taurocholate in male Wistar rats was performed with continuous infusion to prepare the mild AP model (11). The individual microarray results from the mild AP samples are available with the accession numbers GSM1040489, GSM1040490, GSM1040491, GSM1040492, and GSM1040493. Intraductal infusion of sodium taurocholate combined with intermittent infusion and maintaining blood pressure at $60-65 \mathrm{mmHg}$ was performed to prepare the relatively severe AP model. The individual microarray results for the severe AP samples are available with the accession numbers GSM1040484, GSM1040485, GSM1040486, GSM1040487, and GSM1040488. The results of the sham-operated group (control) are available with the accession numbers GSM1040479, GSM1040480, GSM1040481, GSM1040482, and GSM1040483.

Analysis of microarray data. The platform of this experiment was the GPL8786 Affymetrix Multispecies miRNA array (Thermo Fisher Scientific, Inc., Waltham, MA, USA). The Robust Multi-array Average (RMA) algorithm was used to calculate the expression level, and the Microarray Suite algorithm version 5.0 Affymetrix; Thermo Fisher Scientific, Inc.) was used to calculate the detection calls (11). Probes with low expression were filtered and only the groups of samples with at least 2 detection calls were retained. The linear models for microarray data (LIMMA) differential gene screening algorithm was used to screen upregulated and downregulated miRNAs (11). Fold changes were calculated, and miRNAs with a fold change $<0.5$ or $>2$ were considered differentially expressed miRNAs. The P-value cutoff was 0.01 .

Target gene prediction and functional enrichment analysis of miRNAs. The TargetScan (http://www.targetscan.org/), miRanda, (http://www.microrna.org/microrna/home.do), and PicTar (http://pictar.mdc-berlin.de/) programs were used to predict the target genes of the differentially expressed miRNAs. The target genes predicted by the three types of software were screened based on the scoring criteria of each program. In the TargetScan algorithm, target genes with a context percentile $<50$ were excluded. In the miRanda algorithm, target genes with Max-Energy >-10 were excluded. In the PicTar algorithm, target genes with ddG $>-5$ were excluded. The results that were common to all three programs were selected as the final target genes of differential miRNAs.

The predicted target genes of differential miRNAs were used for Gene Ontology (GO) functional annotation (12). Using the Fisher's exact test, enrichment analysis was performed for each GO category. The miRNA-target gene GO network was constructed using the Cytoscape software version 2.8.3 (13).

GO-biological process analysis was performed with the Database for Annotation, Visualization and Integrated Discovery (DAVID) software (david.ncifcrf.gov/), by inputting the list of genes that were predicted to be targets of miR-24 (14).

Patient information. In total, 32 patients were selected for the present study that were admitted into the Department of General Surgery and the ICU ward of Harbin Medical University between July 2015 and December 2015. The inclusion criteria were: Newly admitted patients were assessed within $48 \mathrm{~h}$ and met the diagnostic criteria of the 2012 Atlanta AP guidelines (8). Patients with the following conditions were excluded from the study: Did not agree to participate, were released or died within $24 \mathrm{~h}$ of admission, were younger than 18 years of age, had AP due to pancreatic injury resulting from trauma, had chronic pancreatitis, or had a past history of pancreatitis. According to the severity classification and computed tomography (CT) classification based on the Atlanta international guidelines (15), the included patients were divided into the mild AP group and the moderately severe AP group. Each group included 10 patients. In addition, plasma samples from 10 healthy individuals were used as the control. Clinical information about the patients involved in the present study is listed in Table I. The present study was approved by the Ethics 
Committee of the First Affiliated Hospital of Harbin Medical University (Harbin, China) and conformed to the ethics principles in the Declaration of Helsinki of the World Medical Association (WMA), and the International Ethical Guidelines for Biomedical Research Involving Human Subjects of the Council for International Organizations of Medical Sciences (CIOMS) $(9,16)$. The patient's family and patients agreed to join the experiment and signed an informed consent form. The approval number was HYYKY/WZLS 201560.

Collection of patient's peripheral blood fluid $(10 \mathrm{ml})$. After extraction of blood, the blood was sent to The Central Laboratory of the First Hospital Affiliated to Harbin Medical University within $2 \mathrm{~h}$. Plasma samples were obtained by using a low speed desktop centrifuge (at 3,000 x g for $15 \mathrm{~min}$ at $20^{\circ} \mathrm{C}$ ). The plasma sample was stored at $-80^{\circ} \mathrm{C}$. Blood, urine and calcium analysis was determined by the automatic biochemical apparatus (Beckman AU5800 automatic biochemical analyzer).

Detection of plasma miR-24 in patients using RT-qPCR. Total RNA was extracted from plasma samples using the Ultrapure RNA extraction kit (cat no. CW0581; CWbio Co., Ltd., Beijing, China). A total of $5 \mu \mathrm{g}$ RNA was subjected to $1 \%$ agarose gel electrophoresis to determine the RNA integrity. The reverse transcription of miRNAs was performed using a cDNA first-strand synthesis reagent kit (cat. no. CW2141; CWbio Co., Ltd), according to the manufacturer's instructions (17). The forward primer (5'-3') of miR-24 was TGG CTCAGTTCAGCAGGAACAG and the reverse primer was CTGAGGTGCTGTGCGTGAC. Expression of U6 spliceosomal RNA (U6) was also tested as the internal reference control. The U6 upstream primer was CTCGCTTCGGCA GCACA, and the U6 downstream primer was AACGCT TCACGAATTTGCGT. The PCR cycling conditions were as follows: An initial pre-denaturation step at $95^{\circ} \mathrm{C}$ for $30 \mathrm{sec}$, followed by denaturation at $95^{\circ} \mathrm{C}$ for $5 \mathrm{sec}$, annealing at $60^{\circ} \mathrm{C}$ for $20 \mathrm{sec}$ and extension at $72^{\circ} \mathrm{C}$ for $30 \mathrm{sec}$ for a total of 40 cycles. Fluorescence-based quantification (SYBR Green) was achieved using the Exicycler ${ }^{\mathrm{TM}} 96$ Real-Time Quantitative PCR system. An ABI 7500 fluorescence quantitative PCR machine was used. Relative quantification of the data was performed using the $2^{-\Delta \Delta \mathrm{Cq}}$ method (9).

Statistical analysis. SPSS software version 17.0 (SPSS, Inc., Chicago, IL, USA) was used. The Fisher's exact probability test was performed to compare the differences in gender and age among the three groups. Blood amylase and calcium levels were analyzed using a two-way analysis of variance followed by the Newman-Keuls post hoc test. miR-24 expression results from the patient samples were statistically analyzed using analysis of variance followed by least significant difference test for multiple comparisons. $\mathrm{P}<0.05$ was considered to indicate a statistically significant difference. Each experiment was repeated 3 times independently.

\section{Results}

Differentially expressed miRNAs in activated AR42J cells and in mesenteric lymph samples from AP rats. A gene microarray analysis of activated AR42J cells compared with untreated cells (group 1) was performed in the present study to identify differentially expressed extracellular miRNAs released in the culture media. For a more comprehensive analysis, results from a gene microarray database of mesenteric lymph samples of mild AP (group 2) and severe AP (group 3) were also investigated in the present study. Fig. 1 illustrates the differentially expressed miRNAs comparison between the three groups. The results revealed that several differentially regulated miRNAs were common in more than one group. However, miR-24 was the only miRNA that was significantly and differentially regulated by microarray analysis in all three groups: The activated AR42J cells, the mesenteric lymph in the mild AP rats, and the mesenteric lymph of the severe AP rats (Fig. 1).

Prediction of functions regulated by miR-24. Three programs, TargetScan, miRanda, and PicTar, were used for prediction of the potential target genes of miR-24. Then the potential target genes that were identified in common by all the three programs were used for further GO enrichment analysis, in order to predict the cellular functions that miR-24 may be important in. According to the enrichment index, the most significant functions identified were: 'Positive regulation of calcium-mediated signaling', 'activation of JUN kinase activity', 'calcium ion transport', 'regulation of Rho protein signal transduction', 'negative regulation of protein kinase B signaling cascade', and 'T cell receptor signaling pathway' (Fig. 2). As illustrated in Fig. 3, the predicted miR-24 target genes associated with these functions included vav guanine nucleotide exchange factor 2 (Vav2), spleen associated tyrosine kinase (Syk), luteinizing hormone/choriogonadotropin receptor (Lhcgr), SLC9A3 regulator 1 (Slc9a3r1), calcium channel voltage-dependent beta 1 subunit (Cacnb1), calcium channel voltage-dependent $\mathrm{N}$ type alpha 1B subunit (Cacnalb), B-cell lymphoma/leukemia 10 (Bcl10) and FYVE RhoGEF and PH domain-containing protein $3(\mathrm{Fgd} 3)$.

Clinical characteristics of the patients in the mild AP group, the moderately severe AP group, and the healthy control group. A statistical analysis was performed for the various clinicopathological features of the patients involved in the present study. The results revealed that age and gender did not significantly differ among the control group, the mild AP group, and the moderately severe AP group (Table I). The levels of blood and urine amylases did not significantly differ between the mild AP group and the moderately severe AP group (Table I). However, the calcium levels differed significantly between the mild and severe AP groups $(\mathrm{P}=0.0001$; Table I). According to the CT classification guidelines (15), $\mathrm{CT}$ in the mild AP group indicated that there was no obvious body fluid accumulation, whereas CT in the moderately severe AP group revealed the presence of obvious and large amounts of effusion and even the presence of encapsulated effusion and necrotic tissues (Table I).

miR-24 levels in patients with AP. The miR-24 levels were measured in the plasma of patients in the healthy control group, the mild AP group, and the moderately severe AP group. The results revealed that the mild AP group and the moderately severe AP group had significantly higher expression levels of 
Table I. Clinical characteristics of patients in the mild AP group, moderately severe AP group, and healthy control group.

\begin{tabular}{|c|c|c|c|c|c|}
\hline Indicator & Mild AP group & Moderately severe AP group & Control group & Statistical method & P-value \\
\hline Gender (n) & & & & Fisher's exact test & 0.8792 \\
\hline Female & $3(30 \%)$ & $2(20 \%)$ & $4(40 \%)$ & & \\
\hline Male & $7(70 \%)$ & $8(80 \%)$ & $6(60 \%)$ & & \\
\hline Total & 10 & 10 & 10 & & \\
\hline Age (years) & $47.90 \pm 17.95$ & $47.10 \pm 15.79$ & $44.00 \pm 12.96$ & Fisher's exact test & 0.8428 \\
\hline Blood amylase $(\mu / 1)$ & $491.28 \pm 471.03$ & $413.78 \pm 437.51$ & & 0.11 (t-value) & 0.9165 \\
\hline Urine amylase $(\mu / 1)$ & $2505.60(1160-5346)$ & $3957.90(305-6000)$ & & 0.2656 (z-value) & 0.7906 \\
\hline Calcium (mmol/l) & $2.241 \pm 0.2002$ & $1.74 \pm 0.262$ & & 4.8 (t-value) & 0.0001 \\
\hline
\end{tabular}

CT classification

$\begin{array}{ccc}\mathrm{a} & 0 & 0 \\ \mathrm{~b} & 1 & 0 \\ \mathrm{c} & 9 & 0 \\ \mathrm{~d} & 0 & 0 \\ \mathrm{e} & 0 & 10\end{array}$

Values are presented as means \pm standard deviation, except for the urine amylase that is presented as the median (lower limit of quartile - upper limit of quartile). Bold font denotes a statistically significant result. AP, acute pancreatitis; CT, computed tomography.

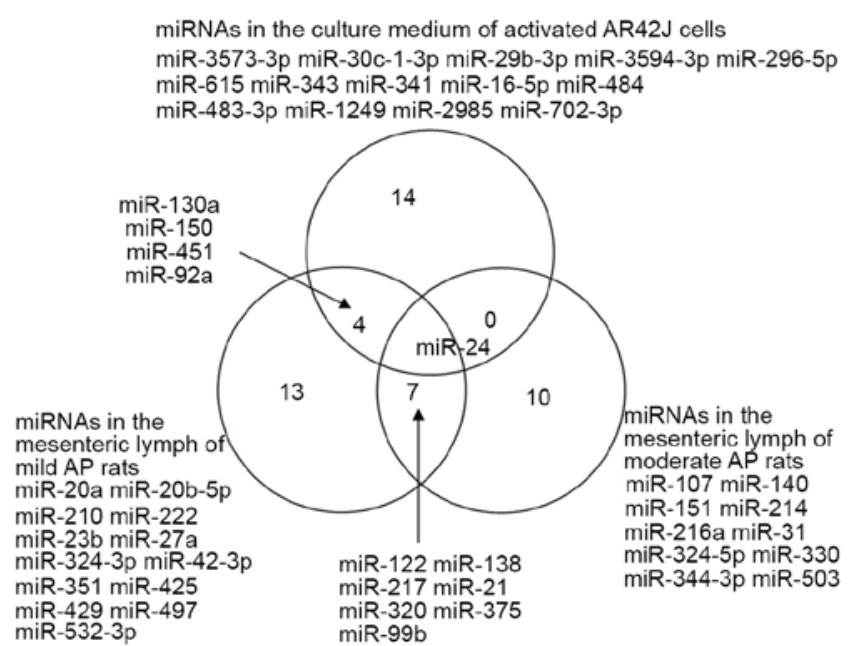

Figure 1. Venn diagram demonstrating the common miRNAs differentially expressed in the three groups analyzed in the present study. miRNA, microRNA; AP, acute pancreatitis.

miR-24 compared with the control group (Fig. 4). However, there was no difference between the mild AP group and the moderately severe AP group (Fig. 4).

\section{Discussion}

This study screened the extracellular miRNA profile in the culture medium of activated rat pancreatic acinar AR42J cells and of the mesenteric lymph of AP rats and discovered that miR-24 was differentially expressed in both groups with high expression. In addition, analysis of expression by RT-qPCR in plasma samples of clinical AP patients demonstrated that miR-24 was significantly upregulated in both the mild AP group and the moderately severe AP

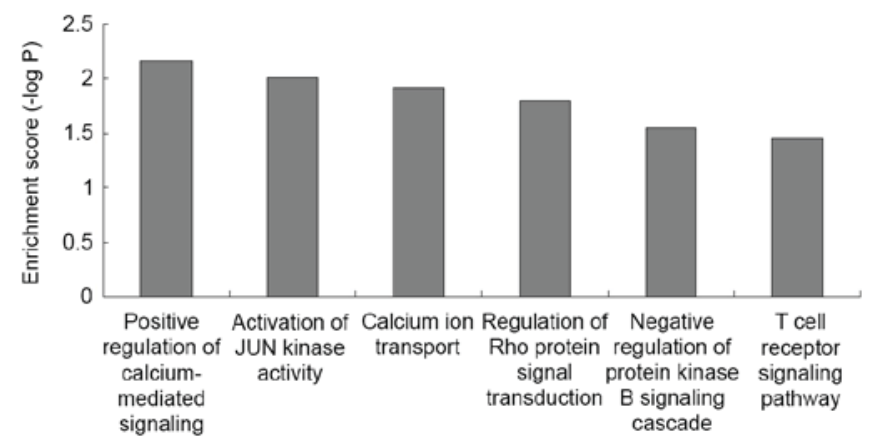

Figure 2. Enrichment analysis of predicted functions for miR-24. Gene ontology-biological process analysis was performed with the DAVID software, by using as input the list of genes that were predicted to be targets of miR-24.

group compared with healthy individuals. These results indicated that during the development of AP, miR-24 might be released in excess into circulating blood. Combined with the findings of a previous study on AP and distant organ injury (18), the present results suggested that miR-24 might have an important role in the process of AP-induced distant organ dysfunction.

Extracellular miRNAs have gradually become a focus of intense study in the medical field $(19,20)$. In 2008, Jeyasee et al first discovered the presence of miRNAs in circulating blood, and since then miRNAs have been demonstrated to be stably present in various extracellular body fluids, including plasma, serum, urine, tears, and lymph $(11,21,22)$. Extracellular miRNAs could integrate into recipient cells and interfere with target mRNAs to regulate the function of recipient cells (23-25). The miRNAs in circulating plasma or serum are packaged into microvesicles and exosomes. Exosomes are small endocytic vesicles, $30-100 \mathrm{~nm}$ in size. They are 


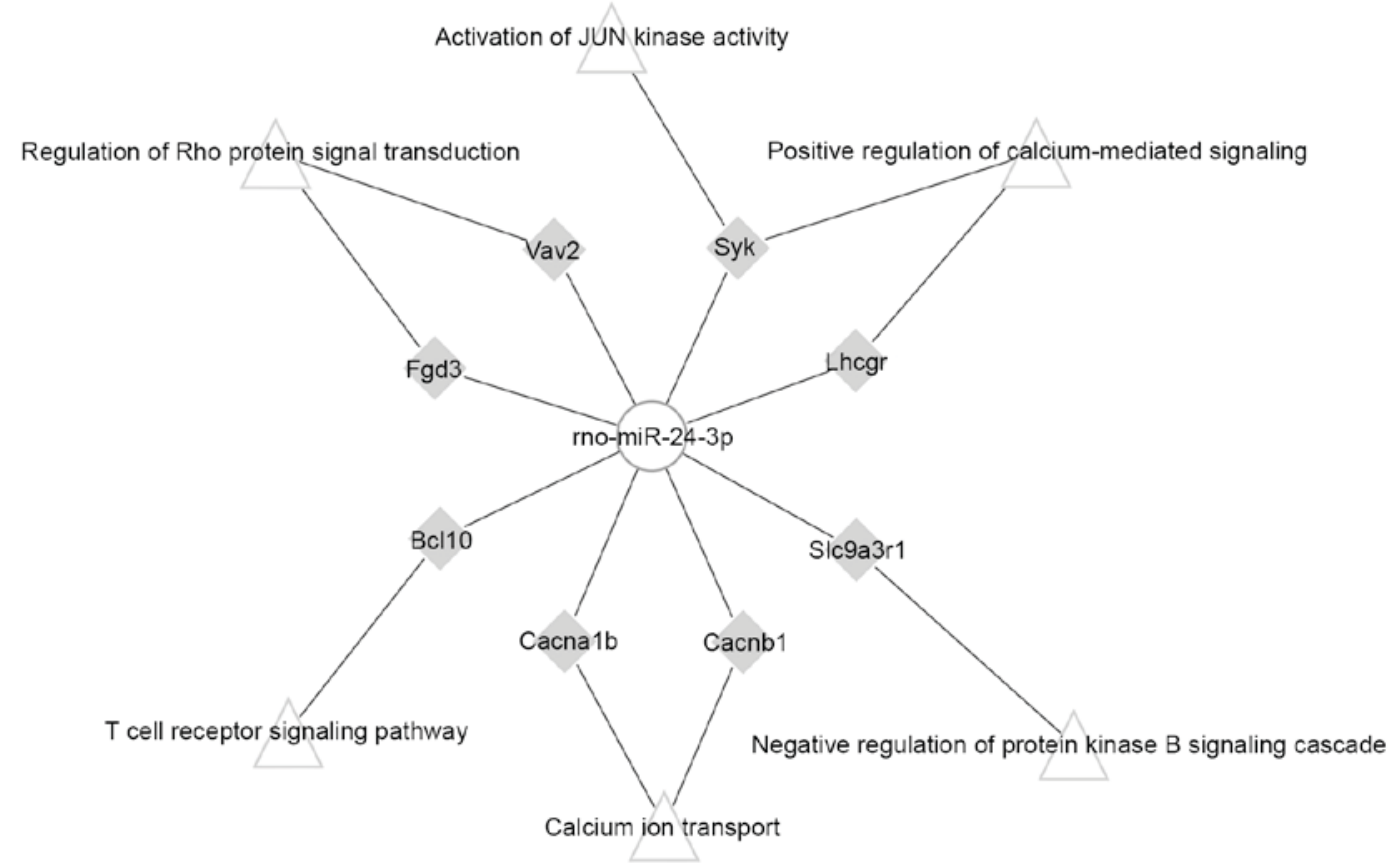

Figure 3. The relationship network of the predicted miR-24 target genes and their functions. Vav2, vav guanine nucleotide exchange factor 2; Syk, spleen associated tyrosine kinase; Lhcgr, luteinizing hormone/choriogonadotropin receptor; Slc9a3r1, SLC9A3 regulator 1; Cacnb1, calcium channel voltage-dependent beta 1 subunit; Cacnalb, calcium channel voltage-dependent $\mathrm{N}$ type alpha 1B subunit; Bcl10, B-cell lymphoma/leukemia 10; Fgd3, FYVE RhoGEF and PH domain-containing protein 3.

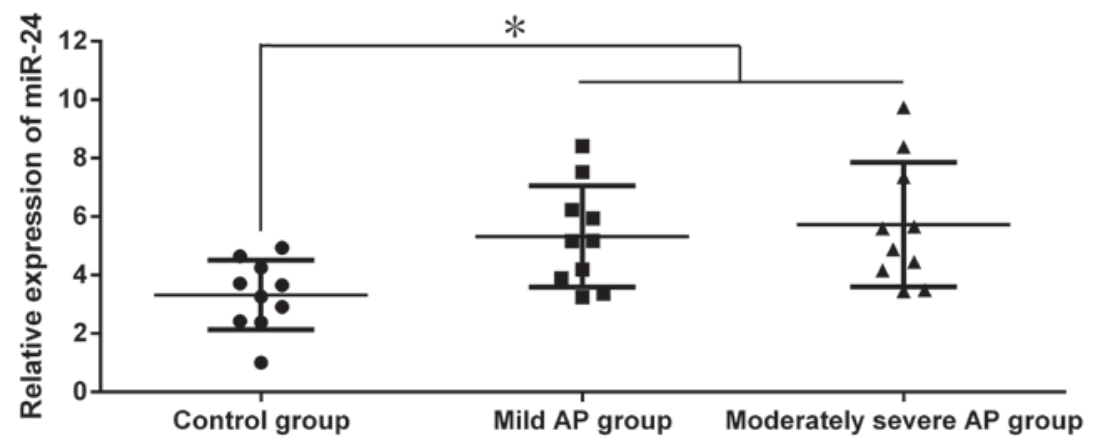

Figure 4. Validation of miR-24 expression in patients with AP. Expression levels of miR-24 relative to U6 internal control were evaluated in plasma samples from patients with mild or moderately severe AP and in control healthy subjects. AP, acute pancreatitis. ${ }^{*} \mathrm{P}<0.05$.

released into the extracellular environment and can fuse with the plasma membrane of recipient cells to induce signal transduction $(26,27)$. Valadi et al $(28)$ prepared total RNA from exosomes and discovered small RNAs, including miRNAs. Hunter et al (29) reported that miRNAs were also expressed in microvesicles in circulating plasma and peripheral blood mononuclear cells in normal individuals (29). Extracellular miRNAs may be useful as novel biomarkers for diagnostic and prognostic purposes and as potential novel therapeutic targets (30.31).

Previous studies on AP and miRNAs rarely evaluated the mechanism and treatment of distant organ injury. Anatomically, mesenteric lymph nodes reach the subclavian vein through the thoracic duct and bypass the portal vein and liver. It is generally considered that potential toxic factors influence other internal organs through mesenteric lymph nodes and bypass the portal vein and liver (32). A previous study has indicated that the intestinal ischemia/reperfusion injury caused by the release of unknown factors into mesenteric lymph nodes could aggravate AP (33). Several studies have suggested that the significantly and specifically upregulated miRNAs in pancreatic tissues, including miR-216a, miR-216b, and miR-375, had pancreatic tissue specificity. The miRNA results from the mesenteric lymph samples of AP mice revealed miR-216a, miR-375 and miR-217 to be significantly upregulated $(11,34)$. These results indicated that mesenteric lymph is likely to contain miRNAs derived from pancreas. Therefore, miRNAs that were present in both pancreatic acini and mesenteric lymph had the greatest possibility of serving a role in the process of the distant organ injury caused by AP or in potential treatments through regulation of signaling pathways.

Furthermore, systemic inflammatory response syndrome (SIRS) occurs in combination with compensatory anti-inflammatory response syndrome (CARS) in AP. In the process of 
inflammatory reactions, anti-inflammatory therapy with the combined inhibition of multiple pro-inflammatory factors has been proven to be effective. For example, treatment using an interleukin (IL)-10 receptor agonist and an anti-IL-8 antibody showed beneficial effects, and antagonizing intercellular adhesion molecule-1 could attenuate lung injury in mice (30). Although the association between pancreatic nuclear factor $(\mathrm{NF})-\kappa \mathrm{B}$ and the activation of trypsinogen in AP is still controversial, a recent study indicated that these processes could both induce inflammation (35); therefore, a combined therapy to inhibit pro-inflammatory responses might be more useful (35). The predominant pathological state of patients with severe AP might be altered from SIRS to CARS by successful treatment (36). The endogenous anti-inflammatory lipid mediator resolvin D1 in AP could improve pancreatic injury and lung injury through the inhibition of NF- $\kappa \mathrm{B}$ activation (37). Circulating miRNAs serving as mediators of intercellular signal transduction have also been confirmed to serve an anti-inflammatory role. Hyaluronic acid-poly (ethyleneimine) nanoparticles that encapsulated miR-223 could regulate the repolarization of pro-inflammatory M1 macrophages to anti-inflammatory M2 macrophages (38). The IL-10-dependent miR-146a exerted anti-inflammatory effects through the Toll-like receptor-4 pathway in monocytes (39). Therefore, it is foreseeable that miRNAs with anti-inflammatory activity have potential significance in the treatment of AP and the control of distant organ injury.

The present study screened miRNAs in the culture media of activated pancreatic acinar cells and the lymph of an AP animal model and revealed that miR-24 was the miRNA that was significantly upregulated in both. Through functional enrichment analysis, significant functions related to miR-24 were predicted: 'Positive regulation of calcium-mediated signaling', 'activation of JUN kinase activity', 'calcium ion transport', 'regulation of Rho protein signal transduction', 'negative regulation of protein kinase B signaling cascade', and ' $\mathrm{T}$ cell receptor signaling pathway'. Previous studies suggested that the dose-dependent inhibition of Rho-kinase had protective effects on AP, whereas bee venom could prevent AP and inhibit pancreatic acinar cell death through the inhibition of c-Jun N-terminal kinase activation $(40,41)$. The phosphoinositide 3-kinase and protein kinase B signal transduction pathways may participate in the pathological process of lung injury in severe AP through the upregulation of $N F-\kappa B$, tumor necrosis factor- $\alpha$, and IL-1 $\beta$ (18). In addition, calcium is important in the process of substance P (SP)-induced chemokine synthesis in pancreatic acinar cells (42). Drug treatment targeting the SP-calcium-mediated signaling pathway might be beneficial for AP therapy. The upregulation of the expression of the sarcoplasmic reticulum calcium pump in pancreatic tissues to reduce intracellular calcium overload could also reduce pancreatic tissue lesions (43).

In the present study, when assaying the plasma of clinical AP patients, we demonstrated that plasma miR-24 levels were significantly higher in patients with mild AP group and moderately severe AP compared with the healthy volunteer control group. These results indicated that CARS was present during the systemic inflammatory reaction of AP, which was consistent with the results from another study (40). However, if the underlying mechanism of miRNA24 in the process of inflammation is further researched, novel therapeutic targets may be found for the treatment of AP, especially for remote organ damage.

Previous studies on miR-24 also indicated a role in inflammatory reactions, anti-inflammatory processes, and apoptosis. Specifically, miR-24 inhibits atherosclerosis and increases macrophage accumulation. Biological analysis indicated that miRNA-24 regulated macrophage activation and polarization. Overexpression of miR-24 inhibited cytokine secretion and exhibited anti-inflammatory functions $(24,44)$. Increasing the miR-24 levels induces cell apoptosis by reducing the Bcl-2 protein expression levels, whereas anti-miR-24 transfection enhances the inflammatory process and cell apoptosis-related reactions $(31,45)$. These findings are consistent with the miR-24 function predicted in the present study. Further studies are required to elucidate the mechanism of miR-24 and its potential as a treatment target.

Although experimental studies related to AP and miRNAs were performed previously, most previous studies evaluated plasma miRNAs only in AP patients to screen for better diagnosis and prediction markers. The novelty of the present study was the discovery of the uniquely expressed extracellular miR-24 in all models tested: activated pancreatic acinar cells, mesenteric lymph from an AP rat model, and plasma samples from patients with AP. In addition, target gene prediction was performed to understand the major cellular functions that miR-24 may be involved in. The present results suggested that miR-24 might be of use as a treatment target in AP and distant organ injury. Further studies on the functions of miRNAs in AP-related inflammatory pathways and mechanisms might facilitate the search for novel therapeutic targets for treating $\mathrm{AP}$ and the related distant organ injury.

\section{Acknowledgements}

This work was supported by the National Natural Science Foundation of China (grant no. 81570579). The authors thank Fenghe Inc. (Shanghai, China) for help in bioinformatics analysis.

\section{References}

1. An F, Zhan Q, Xia M, Jiang L, Lu G, Huang M, Guo J and Liu S: From moderately severe to severe hypertriglyceridemia induced acute pancreatitis: Circulating miRNAs play role as potential biomarkers. PLoS One 9: e111058, 2014.

2. Goodwin D, Rosenzweig B, Zhang J, Xu L, Stewart S, Thompson K and Rouse R: Evaluation of miR-216a and miR-217 as potential biomarkers of acute pancreatic injury in rats and mice. Biomarkers 19: 517-529, 2014.

3. Qin T, Fu Q, Pan YF, Liu CJ, Wang YZ, Hu MX, Tang Q and Zhang HW: Expressions of miR-22 and miR-135a in acute pancreatitis. J Huazhong Univ Sci Technolog Med Sci 34: 225-233, 2014.

4. Huan C, Kim D, Ou P, Alfonso A and Stanek A: Mechanisms of interleukin-22's beneficial effects in acute pancreatitis. World J Gastrointest Pathophysiol 7: 108-116, 2016.

5. Liang X, Zhang B, Chen Q, Zhang J, Lei B, Li B, Wei Y, Zhai R, Liang Z, He S and Tang B: The mechanism underlying alpinetin-mediated alleviation of pancreatitis-associated lung injury through upregulating aquaporin-1. Drug Des Devel Ther 10: 841-850, 2016.

6. Shi C, Andersson R, Zhao X and Wang X: Potential role of reactive oxygen species in pancreatitis-associated multiple organ dysfunction. Pancreatology 5: 492-500, 2005. 
7. Araki Y, Andoh A, Yokono T, Asano N, Yoshikawa K, Bamba S, Ishizuka I and Fujiyama Y: The free radical scavenger edaravone suppresses experimental closed duodenal loop-induced acute pancreatitis in rats. Int J Mol Med 12: 121-124, 2003.

8. Wang H, Jiang Y, Lu M, Sun B, Qiao X, Xue D and Zhang W: STX12 lncRNA/miR-148a/SMAD5 participate in the regulation of pancreatic stellate cell activation through a mechanism involving competing endogenous RNA. Pancreatology 17: 237-246, 2017.

9. Zhao Y, Wang H, Lu M, Qiao X, Sun B, Zhang W and Xue D: Pancreatic acinar cells employ miRNAs as mediators of intercellular communication to participate in the regulation of pancreatitis-associated macrophage activation. Mediators Inflamm 2016: 6340457, 2016

10. Bolstad BM, Irizarry RA, Astrand M and Speed TP: A comparison of normalization methods for high density oligonucleotide array data based on variance and bias. Bioinformatics 19: 185-193, 2003.

11. Blenkiron C, Askelund KJ, Shanbhag ST, Chakraborty M, Petrov MS, Delahunt B, Windsor JA and Phillips AR: MicroRNAs in mesenteric lymph and plasma during acute pancreatitis. Ann Surg 260: 341-347, 2014.

12. Ashburner M, Ball CA, Blake JA, Botstein D, Butler $H$ Cherry JM, Davis AP, Dolinski K, Dwight SS, Eppig JT, et al: Gene ontology: Tool for the unification of biology. The Gene Ontology Consortium. Nat Genet 25: 25-29, 2000.

13. Shannon P, Markiel A, Ozier O, Baliga NS, Wang JT, Ramage D, Amin N, Schwikowski B and Ideker T: Cytoscape: A software environment for integrated models of biomolecular interaction networks. Genome Res 13: 2498-2504, 2003.

14. Jiao X, Sherman BT, Huang da W, Stephens R, Baseler MW, Lane HC and Lempicki RA: DAVID-WS: A stateful web service to facilitate gene/protein list analysis. Bioinformatics 28 : 1805-1806, 2012

15. Balthazar EJ: Acute pancreatitis: Assessment of severity with clinical and CT evaluation. Radiology 223: 603-613, 2002.

16. Council for International Organizations of Medical Sciences: International ethical guidelines for biomedical research involving human subjects. Bull Med Ethics: 17-23, 2002.

17. Gao X, Gulari E and Zhou X: In situ synthesis of oligonucleotide microarrays. Biopolymers 73: 579-596, 2004.

18. Kang X, Wang LZ, Wang YG, Liu L, Fan ZW, Bai LZ and Lu XG: Expression and significance of phosphatidylinositol 3-kinase/protein kinase B signal transduction pathway in severe acute pancreatitis-associated lung injury. Zhonghua Yi Xue Za Zhi 90: 732-737, 2010 (In Chinese).

19. Usborne AL, Smith AT, Engle SK, Watson DE, Sullivan JM and Walgren JL: Biomarkers of exocrine pancreatic injury in 2 rat acute pancreatitis models. Toxicol Pathol 42: 195-203, 2014.

20. Lee EJ, Gusev Y, Jiang J, Nuovo GJ, Lerner MR, Frankel WL, Morgan DL, Postier RG, Brackett DJ and Schmittgen TD: Expression profiling identifies microRNA signature in pancreatic cancer. Int J Cancer 120: 1046-1054, 2007.

21. Kuśnierz-Cabala B, Nowak E, Sporek M, Kowalik A, Kuźniewski M, Enguita FJ and Stepien E: Serum levels of unique miR-551-5p and endothelial-specific miR-126a-5p allow discrimination of patients in the early phase of acute pancreatitis Pancreatology 15: 344-351, 2015.

22. Jeyaseelan K, Lim KY and Armugam A: MicroRNA expression in the blood and brain of rats subjected to transient focal ischemia by middle cerebral artery occlusion. Stroke 39: 959-966, 2008

23. Hamada S, Masamune A, Kanno A and Shimosegawa T: Comprehensive analysis of serum microRNAs in autoimmune pancreatitis. Digestion 91: 263-271, 2015

24. Di Gregoli K, Jenkins N, Salter R, White S, Newby AC and Johnson JL: MicroRNA-24 regulates macrophage behavior and retards atherosclerosis. Arterioscler Thromb Vasc Biol 34 1990-2000, 2014.

25. Katoh M: Therapeutics targeting angiogenesis: Genetics and epigenetics, extracellular miRNAs and signaling networks (Review). Int J Mol Med 32: 763-767, 2013.

26. Lakkaraju A and Rodriguez-Boulan E: Itinerant exosomes: Emerging roles in cell and tissue polarity. Trends Cell Biol 18: 199-209, 2008
27. Zhang Y, Wu XH, Luo CL, Zhang JM, He BC and Chen G: Interleukin-12-anchored exosomes increase cytotoxicity of $\mathrm{T}$ lymphocytes by reversing the JAK/STAT pathway impaired by tumor-derived exosomes. Int J Mol Med 25: 695-700, 2010

28. Valadi H, Ekström K, Bossios A, Sjostrand M, Lee JJ and Lötvall JO: Exosome-mediated transfer of mRNAs and microRNAs is a novel mechanism of genetic exchange between cells. Nat Cell Biol 9: 654-659, 2007.

29. Hunter MP, Ismail N, Zhang X, Aguda BD, Lee EJ, Yu L, Xiao T, Schafer J, Lee ML, Schmittgen TD, et al: Detection of microRNA expression in human peripheral blood microvesicles. PLoS One 3: e3694, 2008.

30. Kylänpää ML, Repo H and Puolakkainen PA: Inflammation and immunosuppression in severe acute pancreatitis. World J Gastroenterol 16: 2867-2872, 2010.

31. Maegdefessel L, Spin JM, Raaz U, Eken SM, Toh R, Azuma J, Adam M, Nakagami F, Heymann HM, Chernogubova E, et al: miR-24 limits aortic vascular inflammation and murine abdominal aneurysm development. Nat Commun 5: 5214, 2014.

32. Fanous MY, Phillips AJ and Windsor JA: Mesenteric lymph: The bridge to future management of critical illness. JOP 8: 374-399, 2007.

33. Flint RS, Phillips AR, Power SE, Dunbar PR, Brown C, Delahunt B, Cooper GJ and Windsor JA: Acute pancreatitis severity is exacerbated by intestinal ischemia-reperfusion conditioned mesenteric lymph. Surgery 143: 404-413, 2008.

34. Endo K, Weng H, Kito N, Fukushima Y and Iwai N: MiR-216a and miR-216b as markers for acute phased pancreatic injury. Biomed Res 34: 179-188, 2013.

35. Kylanpaa L, Rakonczay Z Jr and O'Reilly DA: The clinical course of acute pancreatitis and the inflammatory mediators that drive it. Int J Inflam 2012: 360685, 2012.

36. Ohmoto $\mathrm{K}$ and Yamamoto S: Serum interleukin-6 and interleukin-10 in patients with acute pancreatitis: Clinical implications. Hepatogastroenterology 52: 990-994, 2005.

37. Liu Y, Zhou D, Long FW, Chen KL, Yang HW, Lv ZY, Zhou B, Peng ZH, Sun XF, Li Y and Zhou ZG: Resolvin D1 protects against inflammation in experimental acute pancreatitis and associated lung injury. Am J Physiol Gastrointest Liver Physiol 310: G303-G309, 2016.

38. Tran TH, Krishnan S and Amiji MM: MicroRNA-223 induced repolarization of peritoneal macrophages using CD44 targeting hyaluronic acid nanoparticles for anti-inflammatory effects. PLoS One 11: e0152024, 2016.

39. Curtale G, Mirolo M, Renzi TA, Rossato M, Bazzoni F and Locati M: Negative regulation of Toll-like receptor 4 signaling by IL-10-dependent microRNA-146b. Proc Natl Acad Sci USA 110: $11499-11504,2013$

40. Awla D, Hartman H, Abdulla $\mathrm{A}$, Zhang $\mathrm{S}$, Rahman M, Regnèr $\mathrm{S}$ and Thorlacius $\mathrm{H}$ : Rho-kinase signalling regulates trypsinogen activation and tissue damage in severe acute pancreatitis. $\mathrm{Br} \mathrm{J}$ Pharmacol 162: 648-658, 2011.

41. Bae GS, Heo KH, Park KC, Choi SB, Jo IJ, Seo SH, Kim DG, Shin JY, Kang DG, Lee HS, et al: Apamin attenuated cerulein-induced acute pancreatitis by inhibition of JNK pathway in mice. Dig Dis Sci 58: 2908-2917, 2013.

42. Ramnath RD, Sun J and Bhatia M: Role of calcium in substance $\mathrm{P}$-induced chemokine synthesis in mouse pancreatic acinar cells. Br J Pharmacol 154: 1339-1348, 2008.

43. Xue P, Deng LH, Zhang ZD, Yang XN, Xia Q, Xiang DK, Huang L and Wan MH: Effect of Chaiqinchengqi decoction on sarco/endoplasmic reticulum $\mathrm{Ca}^{2+}$-ATPase mRNA expression of pancreatic tissues in acute pancreatitis rats. World J Gastroenterol 14: 2343-2348, 2008

44. Fordham JB, Naqvi AR and Nares S: miR-24 regulates macrophage polarization and plasticity. J Clin Cell Immunol 6: pii:362, 2015.

45. Zhu Y, You W, Wang H, Li Y, Qiao N, Shi Y, Zhang C, Bleich D and Han X: MicroRNA-24/MODY gene regulatory pathway mediates pancreatic $\beta$-cell dysfunction. Diabetes 62: 3194-3206, 2013. 